\title{
Despistaje de la infección por el virus de inmunodeficiencia hu- mana en Atención Primaria mediante indicadores de sospecha: estudio DIVAPIS
}

\author{
Screening of infection due to the human immunodeficiency virus in primary \\ healthcare with indicators of suspicion: DIVAPIS study
}

\author{
Antonio Ocampo Hermida', Rebeca Longueira Suárez'1, \\ Ángeles Castro Iglesias², Ángel Asorey Carballeira', Javier De la Fuente Aguado ${ }^{3}$ \\ ${ }^{1}$ Medicina Interna, Hospital Álvaro Cunqueiro (EOXI de Vigo). ${ }^{2}$ Medicina Interna, Complejo hospitalario Universitario de A Coruña (CHUAC). \\ ${ }^{3}$ Medicina Interna, Hospital Povisa (Vigo). España
}

\begin{abstract}
Resumen
Introducción: El diagnóstico precoz de la infección por VIH es fundamental para mejorar el pronóstico de la enfermedad y evitar nuevos contagios. Atención Primaria (AP), al ser el primer nivel asistencial al que suele consultar el paciente, brinda la oportunidad de diagnosticar precozmente a muchos de ellos. El objetivo fue potenciar este diagnóstico mediante la identificación de condiciones indicadoras $(\mathrm{Cl})$ de seropositividad que permitan al profesional sospechar la infección.
\end{abstract}

Métodos: Estudio observacional transversal descriptivo con participación de 89 centros de AP de Galicia (Septiembre 2013-Junio 2015). Se recogieron variables clínicas, analíticas y sociodemográficas de pacientes a los que se le realizaba el test de VIH, tanto por sospecha del médico como por petición del propio individuo.

Resultados: De entre 1080 pacientes incluídos en el estudio DIVAPIS se obtuvieron 19 test positivos, con una prevalencia de nuevos diagnósticos del $1.76 \%$. Las variables que resultaron estadísticamente predictores de seropositividad fueron: ser hombre que tiene sexo con hombres $(p=0,02)$, serología positiva para VHB $(p<0,01)$, serología positiva para VHB y VHC $(p=0,02)$ y muguet $(p=0,02)$.

Conclusiones: El desarrollo de herramientas de trabajo en colaboración con AP para mejorar el grado de sospecha de infección por VIH permitió una alta tasa de nuevos diagnósticos y la identificación de distintas $\mathrm{Cl}$ de seropositividad que nos deben alertar de la posibilidad de infección.

Palabras clave: Diagnóstico precoz de VIH, Atención Primaria, condiciones indicadoras

\section{Introducción}

Disminuir el diagnóstico tardío (DT) de la infección por VIH es uno de los principales retos de la respuesta a la epidemia en la actualidad ${ }^{1}$ Se estima que una tercera parte de individuos infectados desconocen estarlo, lo que conlleva con frecuencia un diagnóstico en fases evolucionadas. Esto implica una peor evolución del paciente y al mismo tiempo la posibilidad de que sean fuente de nuevos contagios². En el año 2010 tanto la Organización Mundial de la Salud (OMS) como el European Centre for Disease Prevention and Control (ECDC), publicaron recomendaciones en relación al diagnóstico de la infección por $\mathrm{VIH}^{3,4}$, propugnando acercar la prueba a toda la población haciendo especial énfasis en las poblaciones de mayor riesgo. A este respecto se han tratado de identificar distintas condiciones clínicas y epidemiológicas del paciente

\begin{abstract}
Introduction: The early diagnosis of HIV infection is essential to improve the prognosis of this entity and avoid new infections. Primary healthcare is the most accesible institution for patients, and for this reason provides the opportunity to identify new cases of HIV. The objective was to enhance this diagnostic by indicator conditions that could help the physician diagnose the infection.

Methods: Cross-sectional observational and descriptive study with the participation of 89 primary healthcare centers in Galician (September 2013- June 2015). Clinical, analytical and sociodemographics variables of patients included in the study, with HIV-test requested by the physician or them, were analized.
\end{abstract}

Results: 19 positive test for HIV-infection between 1080 patients included in DIVAPIS study were found, with a prevalence of new diagnoses of $1.76 \%$. Men who have sex with men, positive serology for HBV $(p<0,01)$, positive serology for HBV and HBC $(p=0,02)$ and muguet $(p=0,02)$.

Conclusions: The development of working tools in colaboration with primary healthcare to improve the suspected of HIV-infection sohwed a high rate of new diagnosis and identified indicator conditions that may us suspect the posibility of the infection.

Keywords: Early diagnosis of HIV, primary health care, indicator conditions.

que podrían sugerir la presencia de infección. A nivel europeo, el estudio HIDES $1^{5}$ identificó 8 variables en las que el despistaje de VIH resultaba coste efectivo, encontrando una prevalencia de infección oculta del 1,8\%. Además los pacientes diagnosticados tras la identificación de estas condiciones presentaban una mediana de CD4 de $400 \mathrm{cell} / \mathrm{\mu l}$, lo que suponía un menor porcentaje de diagnósticos tardíos. En España se estima que el número de personas infectadas por el VIH es de aproximadamente 150.000, lo cual implica una prevalencia del $0,4 \%$ en la población general, y de infección oculta del $0,1 \%{ }^{6}$. Atención primaria (AP), al representar la vía de acceso más frecuente a nuestro sistema sanitario, brinda la oportunidad de diagnosticar de forma precoz a muchos de estos pacientes, por lo que resulta prio- 
Tabla 1. Variables analizadas

\begin{tabular}{|c|c|c|}
\hline ANTECEDENTES & CLINICA ACTUAL & VALORES ANALÍTICOS \\
\hline UDVP/exUDVP & Sd mononucleósico & Trombopenia \\
\hline Sexo sin protección & Pérdida de peso & VHB \\
\hline HSH & Poliadenopatías & VHC \\
\hline Pareja con VIH o con riesgo & Deterioro cognitivo & Lúes \\
\hline Tuberculosis & Tuberculosis & Displasia cervical-VPH \\
\hline ETS & ETS & Hipergammaglobulinemia \\
\hline Neumonias repetición & Neumonias de repetición & Otras \\
\hline Muguet & Muguet & \\
\hline Herpes zóster & Herpes zóster & \\
\hline Otras & Dermatitis seborreica & \\
\hline & Condilomas & \\
\hline & Otras & \\
\hline
\end{tabular}

ritario establecer herramientas de colaboración con AP que permitan aumentar el índice de sospecha. Además, conocer las condiciones que hacen sospechar a estos especialistas la presencia de infección nos podría ayudar a identificar otras circunstancias infravaloradas en las que deberíamos hacer hincapié para evitar la pérdida de nuevos diagnósticos. Con el objetivo de conocer todas estas variables en nuestra comunidad, se inició un estudio elaborado por la sociedad gallega Interdisciplinaria de SIDA (SOGAISIDA) con la colaboración de las sociedades gallegas de médicos de AP (AGAMFEC, SEMERGEN y SEMG) para el despistaje de infección por VIH en AP con indicadores de sospecha (DIVAPIS).

\section{Material y métodos}

Se trata de un estudio observacional transversal descriptivo y analítico de prueba diagnóstica. La recogida de la información se centró en las siete áreas asistenciales de AP gallega (Santiago, A Coruña, Ferrol, Lugo, Ourense, Pontevedra y Vigo). Se incluyeron 89 centros y participaron 258 médicos de AP. El período de tiempo de estudio fue desde Septiembre 2013 hasta Junio 2015. Se diseñó un sistema de recogida datos online de los pacientes a los que se le solicitaba el test, en el que se incluyeron variables sociodemográficas (edad, sexo, nacionalidad, nivel académico, conductas de riesgo, estudios previos de VIH) y condiciones clínicas y/0 analíticas consideradas indicadores de sospecha (Tabla 1). También se registraron los casos en los que era el propio paciente quién pedía el estudio, y los motivos por los que lo solicitaba. Todos los pacientes firmaron un documento de consentimiento informado.

Para el análisis de las variables dicotómicas se utilizó el test de Chi-Cuadrado o el test de Fisher exacto bilateral según necesidad. Las variables cuantitativas se analizaron utilizando el test de la T de Student o la U de Mann-Whitney. El análisis univariante se realizó mediante regresión logística binaria. Para el análisis estadístico se utilizó el programa informático SPSS 21.
Tabla 2. Descriptivo global

\begin{tabular}{|c|c|}
\hline VARIABLE & RESULTADO \\
\hline Sexo masculino & $54,6 \%$ \\
\hline Edad (mediana) & 37 \\
\hline Extranjero & $7,8 \%$ \\
\hline Estudios universitarios & $29,1 \%$ \\
\hline Desempleo & $46,9 \%$ \\
\hline Resultado test VIH & $1,8 \%$ \\
\hline Test VIH previo & $36,2 \%$ \\
\hline Solicitud por médico & $58,6 \%$ \\
\hline HSH & $1,2 \%$ \\
\hline VHB & $0,7 \%$ \\
\hline VHC & $1,6 \%$ \\
\hline Muguet & $1,3 \%$ \\
\hline
\end{tabular}

Tabla 3. Estratificación de los resultados por sexo

\begin{tabular}{|c|c|c|c|}
\hline VARIABLE & VARÓN & MUJER & P \\
\hline Edad & 39 & 39 & NS \\
\hline Extranjero & $4,9 \%$ & $11,2 \%$ & $<0,001$ \\
\hline Estudios universitarios & $26,9 \%$ & $32,2 \%$ & NS \\
\hline Desempleo & $47,2 \%$ & $46,3 \%$ & NS \\
\hline Resultado test VIH (+) & $2,4 \%$ & $1 \%$ & NS \\
\hline Test VIH previo & $39,7 \%$ & $32 \%$ & 0,009 \\
\hline Solicitud por médico & $56,6 \%$ & $61,3 \%$ & NS \\
\hline Serología positiva VHB & $1,4 \%$ & $0 \%$ & 0,009 \\
\hline Serología positiva VHC & $1,7 \%$ & $1,4 \%$ & NS \\
\hline Muguet & $0,5 \%$ & $0,2 \%$ & NS \\
\hline
\end{tabular}


Tabla 4. Estratificación de los resultados según solicitud del test

\begin{tabular}{|c|c|c|c|}
\hline VARIABLE & PETICIÓN MÉDICO & PETICIÓN PACIENTE & P \\
\hline Sexo masculino & $52,4 \%$ & $57 \%$ & NS \\
\hline Edad & 42,1 & 36,3 & $<0,001$ \\
\hline Extranjero & $6 \%$ & $10,5 \%$ & 0,005 \\
\hline Estudios universitarios & $24,1 \%$ & $36,4 \%$ & $<0,001$ \\
\hline Desempleo & $50,6 \%$ & $41,5 \%$ & 0,005 \\
\hline Resultado test VIH (+) & $1,7 \%$ & $1,8 \%$ & NS \\
\hline Test VIH previo & $29,9 \%$ & $45,2 \%$ & $<0,001$ \\
\hline
\end{tabular}

\section{Resultados}

Desde Septiembre 2013 hasta Junio 2015 se incluyeron 1244 casos, realizándose finalmente el estudio serológico en 1080 pacientes $(86,8 \%)$.

El $54,6 \%$ fueron varones, con una mediana de edad de 37 años (18-91), la mayoría de ellos de nacionalidad española (92,2\%). El 29,1\% habían cursado estudios universitarios, y casi la mitad de los pacientes (46,9\%) estaban desempleados (Tabla 2). En un 58,6\% de los casos (622) la solicitud de la prueba fue por sospecha del médico aunque en una proporción importante $(41,4 \%, 439)$ fue por petición propia del paciente. Los usuarios que pedían el test eran más jóvenes $(p<0,01)$ y tenían un nivel de estudios significativamente superior $(p<0,01)$ que aquéllos en los que lo solicitaba su médico. En las tablas 3, 4 y 5 se muestran los resultados estratificados en función del sexo, de quién solicitó el test y según el resultado de la prueba, respectivamente.

Se obtuvieron 19 test positivos para infección por VIH $(1,76 \%)$, con un porcentaje similar entre grupos: $11(1,74 \%)$ solicitados por el médico y $8(1,79 \%)$ por petición del paciente. Los motivos por los que el médico había sospechado la infección en estos casos fueron: sexo sin protección (4 casos), hombres que tiene sexo con hombres (3 casos), pareja con infección por VIH (1 caso) y antecedente de muguet (1 caso). Las causas alegadas por los pacientes que habían solicitado el estudio fueron: sexo sin protección (3 casos),

Tabla 5. Estratificación de los resultados por resultado del test

\begin{tabular}{|c|c|c|c|}
\hline VARIABLE & TEST POSITIVO & TEST NEGATIVO & $\mathbf{P}$ \\
\hline Sexo masculino & $73,7 \%$ & $54 \%$ & NS \\
\hline Edad & 37,9 & 39,7 & NS \\
\hline Extranjero & $5,3 \%$ & $7,9 \%$ & NS \\
\hline $\begin{array}{c}\text { Estudios } \\
\text { universitarios }\end{array}$ & $29,4 \%$ & $29,2 \%$ & NS \\
\hline Desempleo & $53,8 \%$ & $46,8 \%$ & NS \\
\hline Test VIH previo & $36,1 \%$ & $42,1 \%$ & NS \\
\hline $\begin{array}{c}\text { Solicitud por } \\
\text { médico }\end{array}$ & $42,1 \%$ & $41,4 \%$ & NS \\
\hline HSH & $10,5 \%$ & $1 \%$ & 0,02 \\
VHB & $10,5 \%$ & $0,6 \%$ & 0,008 \\
\hline VHC & $5,3 \%$ & $1,5 \%$ & NS \\
Muguet & $5,3 \%$ & $0,3 \%$ & 0,02 \\
\hline
\end{tabular}

enfermedades de trasmisión sexual (2 casos), miedo a estar infectado (1 caso), curiosidad (1 caso) y por desconfianza de la pareja (1 caso). El diagnóstico se consideró tardío cuando el recuento de CD4 fue inferior a $350 \mathrm{cel} / \mu \mathrm{ll}$, lo cual sucedió en el $41 \%$ de los casos. Más de la mitad (55\%) de los pacientes diagnosticados por sospecha del médico presentaron este recuento inmunológico en contraposición con sólo el $17 \%$ de aquellos individuos que habían solicitado el test. Tras el análisis estadístico los indicadores que resultaron predictores de seropositividad fueron: ser hombre que tiene sexo con hombres $(p=0,02)$, serología positiva para virus de hepatitis $B(p<0,01)$, serología positiva para virus de hepatitis $B$ y $C(p=0,02)$ y muguet $(p=0,02)$. No se encontró asociación con otras variables como la edad, sexo, nacionalidad, actividad laboral, nivel de estudios, tener test de VIH previo, o con quien había solicitado el estudio. Estos resultados se mantuvieron en el estudio mulitvariante.

\section{Discusión}

Las recomendaciones actuales a nivel nacional para el diagnóstico precoz del VIH en el ámbito sanitario ${ }^{7}$ proponen la realización de la prueba en la siguientes situaciones: personas con criterios clínicos compatibles con infección tales como enfermedades definitorias de SIDA (cáncer cervical invasivo, candidiasis esofágica, criptococosis extrapulmonar, etc), condiciones indicadoras asociadas a prevalencia de VIH no diagnosticado $>0,1 \%$ (infección de transmisión sexual, linfoma maligno, cáncer/displasia anal, etc) y otras condiciones en las que la no identificación de la infección puede tener consecuencias negativas importantes para el manejo clínico del paciente (enfermedades que requieren tratamiento inmunosupresor agresivo, lesión cerebral primaria ocupante de espacio, púrpura trombocitopénica idiopática). Se recomienda también a las personas que por su exposición al VIH o su procedencia requieran descartar infección, y de forma obligatoria en donantes de sangre, trasplante, injerto y usuarios relacionados con técnicas de reproducción asistida. Debido a la necesidad de reducir los casos de infección no diagnosticada, en la actualidad se recomienda ofrecer el estudio a todo paciente sexualmente activo entre 2059 años, que requiera extracción de sangre por cualquier 
otro motivo y que resida en provincias con tasas de nuevos diagnósticos de VIH en ese grupo de edad, A este respecto el estudio europeo HIDES $2^{8}$ analizó la adherencia a las recomendaciones de las guías para la realización del test. Los autores encontraron que se solicitó el estudio en sólo un $72 \%$ de los casos a pesar de presentar indicadores de riesgo claramente establecidos, dejando por lo tanto un porcentaje significativo de casos potencialmente no diagnosticados. A nivel nacional Pérez y cols ${ }^{9}$, mediante la aplicación de un sencillo cuestionario en AP y Urgencias basado en exposiciones de riesgo y condiciones indicadoras, describieron una prevalencia de infección oculta del 4,1\% entre los pacientes que reunían 10 más de estas variables, insistiendo en la necesidad de implementar medidas que faciliten el diagnóstico precoz de la infección por VIH. En el trabajo de Cayuelas y cols ${ }^{10}$, tras un estudio multicéntrico de colaboración con AP compuesto de sesiones formativas y la participación en el estudio HIDES, se consiguió triplicar la petición de VIH, con una tasa de infección del 2,3\%. Esta necesidad es una realidad también en nuestra comunidad gallega, en donde un $30 \%$ son diagnósticos tardíos ${ }^{11}$, que en muchas ocasiones ocurren durante un ingreso hospitalario por infecciones oportunistas. Nuestro diseño de recogida de datos on line demostró una buena aceptación por los equipos de AP, consiguiendo tasas de diagnóstico de VIH elevadas (1,76\%), muy similares a las descritas en otros trabajos basados en condiciones indicadoras como el estudio HIDES ${ }^{5}$. De entre las variables analizadas, el antecedente de infección para VHB, coinfección por VHB-VHC, la presencia de muguet y el ser hombre que tiene sexo con hombres, se asociaron de forma significativa con la presencia de VIH, por lo que en nuestro entorno estas condiciones deben alertar al clínico de la posibilidad de infección. Nos parece también destacable el alto porcentaje de peticiones del test por el paciente que encontramos en nuestro estudio, con una proporción de resultados positivos similar al grupo en el que el médico era quien sospechaba la infección. Además, en más de la mitad de los casos en los que el facultativo sospechó la infección el diagnóstico fue tardío, siendo esto mucho menos frecuente cuando el paciente pedía el estudio. En estos casos la solicitud propia del test reflejaría una potencial exposición a situaciones de riesgo identificadas por el individuo, aunque queda patente la necesidad de aumentar el grado de sospecha por parte de los profesionales. Otros trabajos como el de Ayerdi-Aguiberrenbegoa y cols ${ }^{12}$ han mostrado que un porcentaje importante de nuevos diagnósticos tenían un test previo de VIH, lo cual apoya que muchos de estos pacientes eran probablemente conocedores de su situación de alto riesgo. A este respecto, en nuestro estudio no encontramos significación estadística con el hecho de haber realizado anteriormente el test, aunque como limitación del estudio consideramos que es posible que el limitado tamaño de la muestra no sea suficiente para valorar el peso real de todos los marcadores analizados.
En conclusión, en este trabajo el desarrollo de herramientas de trabajo en colaboración con AP para mejorar el diagnóstico precoz del VIH mostró gran aceptación por parte de estos profesionales, permitiendo una elevada tasa de nuevos diagnósticos y permitiendo identificar variables predictoras de seropositividad mediante un método ágil y de fácil cumplimentación. Creemos que la colaboración entre distintos niveles asistenciales como lo reflejado en este estudio ayuda a mejorar el conocimiento de la infección y a aumentar el grado de sospecha, consiguiendo favorecer el diagnóstico temprano. Es fundamental continuar desarrollando estrategias que permitan avanzar en la detección precoz de la infección por VIH para mejorar el pronóstico de estos pacientes y disminuir el riesgo de trasmisión en la población general.

\section{Bibliografía}

1. European Centre for Disease Prevention and Control. HIV/AIDS Surveillance in Europe 2010 Report. ECDC 2010. Disponible en http://ecdc.europa.eu/en/publications/Publications/111129_SUR_Annual_HIV_Report.pdf.

2. Hamers FF, Philips AN. Diagnosed and undiagnosed HIV infected populations in Europe. HIV Med 2008;9:6-12.

3. European Centre for Disease Prevention and Control. HIV testing: increasing uptake and effectiveness in the European Union. ECDC 2010. Disponible en http://ecdc. europa.eu/en/publications/Publications/101129_gui_hiv_testing.PDF.

4. WHO European Region. Scaling up HIV testing and counseling in the WHO European Region as an essential component of efforts to achieve universal acces to HIV prevention, treatment, care and support. Policy framework. WHO/EURO 2010. Disponible en http://www.euro.who.int/data/assets/pdf file/0007/85489/E93715. pdf?ua $=1$

5. Sullivan AK, Raben D, Reekie J, Rayment M, Mocroft A, Esser S et al. Feasibility and effectiveness of indicator condition-guided testing for HIV: results from HIDES I (HIV Indicator Diseases across Europe Study). PLoS one 2013;8:e52845

6. Área de vigilancia de VIH y conductas de riesgo. Vigilancia epidemiológica del VIH/ SIDA en España: sistema de información sobre nuevos diagnósticos de VIH y registro nacional de casos de SIDA. Plan Nacional sobre el SIDA-SG de Promoción de la Salud y Epidemiología-Centro Nacional de Epidemiología-ISCIII 2015. Disponible en http://www.msssi.gob.es/ciudadanos/enfLesiones/enfTransmisibles/sida/vigilancia/ informeVIH_SIDA_2015.pdf

7. Guía de recomendaciones para el diagnóstico precoz de VIH en el ámbito sanitario. Ministerio de Sanidad, Servicios Sociales e Igualdad, Plan Nacional sobre Sida. 2014. Disponible en http://www.msssi.gob.es/ciudadanos/enfLesiones/enfTransmisibles/sida/docs/GUIA_DX_VIH.pdf

8. Raben D, Mocroft A, Rayment M, Mitsura VM, Hadziosmanovic V, Sthoeger ZM et al. Auditing HIV testing rates across Europe: results from the HIDES 2 Study. PLOS One. 2015; 10: e0140845

9. Pérez MJ, Gómez-Ayerbe C, Pérez P, Muriel A, Díaz A, Martínez-Colubi M et al. Development and Validation of an HIV Risk Exposure and Indicator Conditions Questionnaire to Support Targeted HIV Screening. Medicine. 2016;95:e2612.

10. Cayuelas-Redondo L, Menacho-Pascual I, Nogueira-Sánchez P, Goicoa-Gago C, Pollio-Peña G, Blanco-Delgado $R$ et al. Indicator condition guided human immunodeficiency virus requesting in primary health care: results of a collaboration. Enferm Infecc Microbiol Clin. 2015;33:656-662.

11. Dirección Xeral de Saúde Pública. Informe VIH-sida en Galicia 2016 [monografía en internet]. Santiago de Compostela: Xunta de Galicia, Consellería de Sanidade; 2016. Dispoñible en: http://www.sergas.es/saudepublica/informes-epidemioloxicos-de-vih-sida.

12. Rivero A, Moreno S. ¿Es el momento de poner en marcha nuevas estrategias de prevención de la infección por VIH en España?. Enferm Infecc Microbiol Clin 2017;35:271-2. 